\title{
Efeitos da intervenção musical no nível de ansiedade de pacientes em tratamento em um Centro de Atenção Psicossocial do sudeste do Pará
}

\author{
Effects of musical intervention on the anxiety level of patients undergoing treatment in a \\ Psychosocial Care Center in southeastern Pará
}
Efectos de la intervención musical sobre el nivel de ansiedad de los pacientes sometidos a tratamiento en un Centro de Atención Psicosocial en el sureste de Pará

\begin{abstract}
Fernanda Cristina da Costa Silva ${ }^{1}$, Adriana Paiva Camargo Saraiva ${ }^{1 *}$, Rômulo Pinheiro Santos ${ }^{1}$, Maiara Goudim de Melo ${ }^{1}$, Sandra dos Santos Tavares ${ }^{1}$, Joaquim Henrique Ribeiro Pereira ${ }^{1}$, Anny Karoliny Nascimento Carvalho'.
\end{abstract}

\section{RESUMO}

Objetivo: Investigar os efeitos da intervenção musical no nível de ansiedade de pacientes em tratamento em um Centro de Atenção Psicossocial (CAPS) do sudeste do Pará. Métodos: Estudo quase-experimental, aprovado por Comitê de Ética em Pesquisa, envolvendo 10 pacientes do CAPS contando com aplicação de Inventário de Ansiedade, antes e após sessões de intervenção musical. O repertório musical de 10 músicas contou com mensagens positivas e melodias suaves, majoritariamente. Os dados obtidos foram analisados por estatística descritiva com auxílio do software IBM SPSS Statistics. Resultados: A maior proporção dos pacientes tem entre 29 e 36 anos (40\%), sexo feminino (70\%), Ensino Fundamental Incompleto (30,0\%), Ensino Médio Completo $(30,0 \%)$, são solteiros $(60,0 \%)$, tem depressão $(40,0 \%)$, fazem tratamento há pelo menos 6 anos (40,0\%), toma remédios controlados (90\%) e não possui outro problema de saúde $(60 \%)$. Houve redução significativa no nível de ansiedade médio, que passou de 48,60 para 39,30 ( $p=0.0001$ ). Conclusão: A intervenção musical mostrou que pode auxiliar na diminuição da ansiedade de modo significativo em pacientes portadores de transtorno mental. Este estudo é pioneiro no CAPS em questão e a música deve ser estimulada em outros centros.

Palavras-chave: Enfermagem, Música, Ansiedade.

\begin{abstract}
Objective: To investigate the effects of musical intervention on the anxiety level of patients undergoing treatment at a Psychosocial Care Center (CAPS) in southeast Pará. Methods: Quasi-experimental study, approved by the Research Ethics Committee, which involves 10 patients with the application of the Anxiety Inventory, before and after the musical intervention sessions. The 10-song musical repertoire had mostly positive messages and soft melodies. The data obtained was analyzed using descriptive statistics using the IBM SPSS Statistics software. Results: The highest proportion of patients is between 29 and 36 years old $(40 \%)$, women $(70 \%)$, incomplete primary school $(30.0 \%)$, complete secondary school $(30.0 \%)$, are single $(60$, $0 \%)$, have depression $(40.0 \%)$, has been treated for at least 6 years $(40.0 \%)$, takes controlled medications $(90 \%)$, and has no other health problems $(60 \%)$. There was a significant reduction in the mean level of anxiety, which went from 48.60 to 39.30 ( $p=0.0001$ ). Conclusion: Musical intervention showed that it can significantly reduce anxiety in patients with mental disorders. This study is a pioneer in the CAPS in question and music should be promoted in other centers.
\end{abstract}

Key words: Nursing, Music, Anxiety.

1Universidade do Estado do Pará (UEPA), Conceição do Araguaia - PA. * E-mail: adriana.saraiva@uepa.br 


\section{RESUMEN}

Objetivo: Investigar los efectos de la intervención musical sobre el nivel de ansiedad de los pacientes sometidos a tratamiento en un Centro de Atención Psicosocial (CAPS) en el sureste de Pará. Métodos: Estudio cuasiexperimental, aprobado por el Comité de Ética de Investigación, que involucra a 10 pacientes contando con la aplicación del Inventario de Ansiedad, antes y después de las sesiones de intervención musical. El repertorio musical de 10 canciones tenía mensajes principalmente positivos y melodías suaves. Los datos obtenidos se analizaron utilizando estadísticas descriptivas utilizando el software IBM SPSS Statistics. Resultados: La mayor proporción de pacientes tiene entre 29 y 36 años (40\%), mujeres (70\%), escuela primaria incompleta $(30.0 \%)$, escuela secundaria completa $(30.0 \%)$, son solteros $(60,0 \%)$, tiene depresión (40.0\%), ha recibido tratamiento durante al menos 6 años $(40.0 \%)$, toma medicamentos controlados $(90 \%)$ y no tiene ningún otro problema de salud $(60 \%)$. Hubo una reducción significativa en el nivel medio de ansiedad, que pasó de 48,60 a 39,30 ( $p=0,0001$ ). Conclusión: La intervención musical mostró que puede reducir significativamente la ansiedad en pacientes con trastornos mentales. Este estudio es pionero en lo CAPS en cuestión y la música debería fomentarse en otros centros.

Palabras clave: Enfermeria, Musica, Ansiedad.

\section{INTRODUÇÃO}

A música é uma expressão artística utilizada como recurso terapêutico por diversas culturas desde a Antiguidade. Porém, somente no século XIX se teve o primeiro registro do cuidado humanizado com música, por meio da enfermeira Florence Nightingale, que relatou efeitos benéficos da voz humana, instrumentos de sopro e cordas, em soldados feridos durante a guerra da Crimeia. Posteriormente as enfermeiras musicistas norte americanas Isa Maud Ilsen e Harriet Ayer Seymour fizeram utilização desse recurso para aliviar a dor física e emocional dos soldados feridos durante a I e a II Guerra Mundial (FRANZOI MAH, et al., 2016; MIRANDA MC, et al., 2017; BATISTA EC, et al., 2018).

Batista NS e Ribeiro MC (2016) ressaltam que as emoções podem influenciar e serem influenciadas pelos processos sofridos no corpo e ainda, que existe uma relação indissociável entre corpo e mente no que se refere à dor crônica. De acordo com Almeida AS e Silva MR (2013), a música produz emoções e para isso, seus elementos, como melodia e timbre, são processados no sistema límbico do cérebro. As emoções do ouvinte também podem ser alteradas pela ativação de uma memória passada que a música provoca no indivíduo (CHAN MF, et al., 2012).

Nunes-Silva M, et al. (2016) e Areias JC (2016) defendem que a música promove a redução da ansiedade. A ansiedade é definida como "um sentimento de medo vago e desagradável caracterizado por um desconforto ou tensão derivado de uma antecipação de perigo, de algo desconhecido ou estranho". Essa condição passa a ser patológica quando ocorre de forma exagerada, desproporcional ao estímulo, resultando em uma alteração na qualidade de vida do indivíduo (CASTILLO ARG, et al., 2000).

Firmeza MA, et al. (2017) ressaltam o potencial ansiolítico da música, devido ao efeito que ela exerce sobre as vias dopaminérgicas mesolímbicas. Sendo assim, pode ser considerada como recurso terapêutico, sem efeitos colaterais e de baixo custo. A música também reduz o estresse e causa relaxamento. Esses efeitos estão relacionados à da escuta musical na região frontal esquerda do cérebro, ativando o sistema límbico, centro das emoções, provocando a liberação de neurotransmissores como noradrenalina, serotonina, dopamina e endorfina, ligados ao bem-estar, que é estendido ao corpo, desencadeando o relaxamento de fibras musculares (ARAÚJO TC e SILVA LWS, 2013).

Rohr RV e Alvim NAT (2016) destacam outros efeitos benéficos como: promoção da comunicação, interação, empatia, vínculo, diálogo, expressão de emoções e sentimentos, estímulo de funções cognitivas e aprendizado, reflexão sobre o cuidado, melhora da autoestima e redução da dor.

Profissionais de saúde de um modo geral podem utilizar a música, desde que seja na forma de intervenção musical. Os termos musicoterapia e intervenção musical se assemelham, porém, tem diferenças. A musicoterapia é de uso privativo do profissional musicoterapeuta que faz uso de técnicas e métodos específicos; já a intervenção musical ou música, em medicina, pode ser exercida por demais profissionais de saúde como terapia complementar (FRANZOI MAH, et al., 2016). 
Franzoi MAH, et al. (2016) incentiva a utilização da música por enfermeiros na, uma vez que esta está inserida na Classificação de Intervenções de Enfermagem - Nursing Intervention Classification (NIC). Em 2010 o Conselho Regional de Enfermagem de São Paulo (COREn-SP) deu respaldo a esses profissionais para a utilização da música, por meio do uso da voz ou aparelho de som, "basta que tenha sensibilidade para reconhecer as potencialidades da música no cuidado de enfermagem" (ROHR RV e ALVIM NAT, 2016).

De acordo com Batista NS e Ribeiro MC (2016) a música também pode ser utilizada como terapia complementar no cuidado a pessoas em sofrimentos psíquico, para promover a inclusão e reabilitação das mesmas. Contudo, nem sempre essas pessoas receberam um cuidado que visasse a sua inclusão e reabilitação, pois durante um longo período da história, indivíduos com transtorno mentais foram cuidados por familiares, abandonadas nas ruas ou em hospitais psiquiátricos, onde nem sempre recebiam uma forma de tratamento adequada (BOTELHO JV e LIMA MV, 2015).

O modelo hospitalocêntrico, bem como a assistência de enfermagem ofertada aos pacientes nos manicômios passou a ser criticada na década de 1970, o que culminou na efetivação e expansão de um movimento político na área da saúde conhecido como Reforma Psiquiátrica na década de 1980. Essa reforma norteou a criação da Política Nacional de Saúde Mental instituída em 2001, que reverteu o modelo manicomial no modelo de atenção psicossocial (ESPERIDIÃO E, et al., 2013).

A Política Nacional de Saúde Mental, passa a priorizar os Centros de Atenção Psicossocial (CAPS) na assistência de pessoas com transtornos mentais em substituição às internações hospitalares. Esses centros funcionam em locais de caráter aberto e comunitário, com três modalidades de tratamento: intensivo (diário); semi-intensivo (três vezes por semana, aproximadamente) e não intensivo (periodicidade semanal). Cada centro é constituído por uma equipe multiprofissional, que atua sob a ótica multidisciplinar. A reabilitação psicossocial é facilitada pelas oficinas terapêuticas, que são estratégias inseridas como uma das formas de tratamento nesses centros, de acordo com o modelo de assistência à saúde mental proposto pela Lei $n^{\circ}$ 10.216, de 6 de abril de 2001 (BRASIL, 2004).

As oficinas terapêuticas podem ser oficinas expressivas contendo teatro, fotografia, expressão plástica, expressão verbal, expressão corporal e expressão musical. Nas oficinas de expressão musical, a música proporciona a diminuição da ansiedade, possibilita integração e inserção dos pacientes, desenvolvimento de ressocialização, cultivo de amizades e a diminuição de angústias e confusão existencial (MENDES MF, et al., 2016; NASCIMENTO E, et al., 2018)

Apesar dos efeitos positivos que a música proporciona a pacientes em sofrimento psíquico, pode haver efeitos negativos se utilizada de forma indiscriminada, oferecendo o risco de estresse, desconforto, sofrimento e agravamento de comportamentos típicos dos transtornos que apresentam (GATTINO GS, 2016).

Sabendo que no sudeste do Pará não há relatos de estudos de intervenção musical junto aos portadores de transtornos mentais em tratamento no CAPS, justifica-se realizar um estudo com objetivo de investigar os efeitos da intervenção musical como estratégia do cuidado de enfermagem em um CAPS do sudeste do Pará.

\section{MÉTODOS}

Trata-se de um estudo quase-experimental, aprovado pelo Comitê de Ética em Pesquisa (CEP) envolvendo seres humanos, parecer no 3.398.267, realizado no CAPS de um município do sudeste do Pará, durante o período de 25 de setembro a 25 de outubro de 2019. Este centro conta com uma equipe de saúde composta por uma enfermeira, uma psicóloga, um médico clínico geral, um psiquiatra, uma assistente social, uma terapeuta ocupacional e uma técnica de enfermagem. O local foi escolhido por ser o único centro de tratamento de pessoas com transtornos mentais do município em estudo, onde são atendidos diariamente cerca de 30 pacientes, sendo que, aproximadamente, a metade deles participa de forma regular das atividades em grupo promovidas por este centro.

A amostra incluiu 10 pacientes maiores de 18 anos que participam regularmente de atividades em grupo e que aceitaram participar do estudo, por meio da assinatura do Termo de Consentimento Livre e Esclarecido (TCLE). A autonomia dos participantes foi certificada pela psicóloga do referido centro. Entre os participantes, estão portadores depressão, síndrome do pânico, transtorno afetivo bipolar, esquizofrenia e transtorno de 
ansiedade. Não foram incluídos pacientes que necessitam de um tutor ou responsável legal, por exemplo, portadores de demência. Porém, os pacientes que não foram incluídos e estavam presentes em algum momento da intervenção musical, Ihes foi facultada a participação na qualidade de ouvinte, com a presença da psicóloga do CAPS.

Inicialmente, ocorreu o planejamento da intervenção musical. As músicas foram selecionadas a partir da análise da letra da música, que incluía uma mensagem positiva, otimista, encorajadora; temas universais como o amor, natureza ou pela possibilidade de sugestão de equilíbrio e harmonia (TORCHI TS e BARBOSA MAM, 2006). A partir do aval da psicóloga do CAPS de um repertório de 10 músicas, foi elaborado o cronograma das atividades da intervenção e com a aprovação da coordenação do CAPS, ocorreu a primeira abordagem dos pacientes para apresentar o estudo e convidá-los para participar.

Após assinatura do TCLE, cada participante foi abordado de forma individual para responder a um questionário sociodemográfico e terapêutico composto por 9 questões e receber o cronograma das atividades da intervenção musical.

A psicóloga da instituição esteve presente na aplicação dos instrumentos de coleta de dados e nas atividades musicais. Por ter acesso aos dados pessoais dos pacientes, ela recebeu um cartão de frequência de cada participante da pesquisa, no qual ela conhecia os participantes também pela identificação alfanumérica e registrou a presença deles em cada encontro. Ao final do estudo ela repassou a frequência de cada participante contendo apenas a identificação alfanumérica para a equipe de pesquisa.

Foram realizadas 15 sessões de intervenção musical com duração de 30 a 60 minutos cada, que foram inseridas nas atividades regulares do CAPS com a participação da equipe multiprofissional. Os participantes responderam ao Inventário de Ansiedade Traço Estado (IDATE), instrumento validado, traduzido e adaptado para o português, que tem por objetivo avaliar a ansiedade, e é considerado um instrumento adequado para avaliar o nível de ansiedade transitória de pessoas sob cuidados psiquiátricos. Segundo Borine MS (2011), esse instrumento pode ser utilizando tanto para pessoas adultas normais, quanto para pessoas com transtornos mentais.

O IDATE foi respondido antes e no final da primeira sessão de intervenção musical e no final da última sessão, para comparar o nível de estresse antes e depois da primeira sessão e depois da última sessão. $O$ intuito foi verificar o impacto da intervenção de forma imediata e mediata após uma série de sessões musicais. Considerando que os resultados desse inventário poderiam ser de interesse da psicóloga do CAPS, eles foram compartilhados com a mesma como forma de beneficiar os pacientes para avaliação e tratamento precoce de transtornos de ansiedade.

Os dados coletados foram tabulados, interpretados, processados e analisados por meio da estatística descritiva e inferencial, com nível de significância de $p$-valor $<0.05$. Foi realizado o teste da razão de verossimilhança do Qui-quadrado para amostras independentes (razão de probabilidade), indicado em caso de pequenas amostras.

Para verificar o nível de ansiedade dos participantes antes e depois da intervenção foi realizado o teste $t$ de Student para comparação das médias obtidas nos diferentes momentos. Para a análise dos dados foram utilizados recursos de computação, por meio do processamento no sistema Microsoft Excel, Statistic Package for Social Sciences (SPSS) versão 24.0, todos em ambiente Windows 7.

\section{RESULTADOS}

O repertório com 10 músicas, incluiu: "O Sol" - Jota Queste; "É preciso saber viver" - Titãs; "Peça felicidade" - Melim; "Jardineira" - Orlando Silva; "Onda diferente" - Anitta - Adaptação e "Meu abrigo" Melim - Adaptação; e as seguintes músicas autorais: "O importante é nunca desistir"; "Pra viver bem"; "O dia mau vai passar"; "Pagar o mal com o bem".

A caracterização da amostra, quanto aos dados sociodemográficos e terapêuticos, mostra, conforme a Tabela 1, que a maior proporção dos pacientes em tratamento neste CAPS possui idade entre 29 e 36 (4; $40 \%$ ), sexo feminino (7; 70\%), possuem o Ensino Fundamental Incompleto (3; 30,0\%), e Ensino Médio Completo $(3 ; 30,0 \%)$ e são solteiros $(6 ; 60,0 \%)$ (Tabela 1 ). 
Tabela 1 - Distribuição dos pacientes em tratamento em um Centro de Atenção Psicossocial (CAPS) do sudeste do Pará, segundo a caracterização sociodemográfica.

\begin{tabular}{lccc}
\hline Caracterização Sociodemográfica & Quantidade & Percentual (\%) & P-Valor $^{(1)}$ \\
\hline Faixa Etária & & & \\
\hline $29-36$ & 4 & $40,0 \%$ & \\
$37-44$ & 2 & $20,0 \%$ & $0.002^{*}$ \\
$45-52$ & 2 & $20,0 \%$ & \\
$53-60$ & 2 & $20,0 \%$ & \\
\hline Gênero & & & \\
\hline Feminino & 7 & $70,0 \%$ & \multirow{2}{*}{$0.0001^{*}$} \\
Masculino & 3 & $30,0 \%$ & \\
\hline Escolaridade & & & \\
\hline Ensino Fundamental Incompleto & 3 & $30,0 \%$ & \\
Ensino Médio Completo & 3 & $30,0 \%$ & \\
Ensino Médio Incompleto & 1 & $10,0 \%$ & $0.736 \mathrm{~ns}$ \\
Ensino Superior & 2 & $20,0 \%$ & \\
Nunca Estudou & 1 & $10,0 \%$ & \\
\hline Estado Civil & \multicolumn{3}{c}{} \\
\hline Casada & 3 & $30,0 \%$ & \\
Divorciada & 1 & $10,0 \%$ & $0.024^{*}$ \\
Solteiro & 6 & $60,0 \%$ & \\
\hline
\end{tabular}

Legenda: (1) Teste Qui-quadrado de Pearson (p-valor <0.05). *Valores Significativos; NS Valores Não Significativos. $H_{0}$ : Não há tendência significativa entre as frequências observadas $(p>0.05)$. $H_{1}$ : Há tendência significativa entre as frequências observadas $(p<0.05)$.

Fonte: SILVA FCC et al., 2020.

A Figura 1 mostra que os pacientes possuem idade média igual a 43 anos $(\mu \approx 42.7)$, variando com desvio padrão de $\pm 9.91 \approx 10$ anos. A variação da idade dos pacientes se mostra elevada (Var $=98.23)$. A idade mínima dos pacientes é 29 anos e a máxima é 57 anos. A mediana indica que $50 \%$ dos pacientes possuem idade acima de 41 anos (Figura 1).

Figura 1 - Idade média dos pacientes em tratamento em um Centro de Atenção Psicossocial (CAPS) do sudeste do Pará.

IDADE MÉdIA DOS PACIENTES
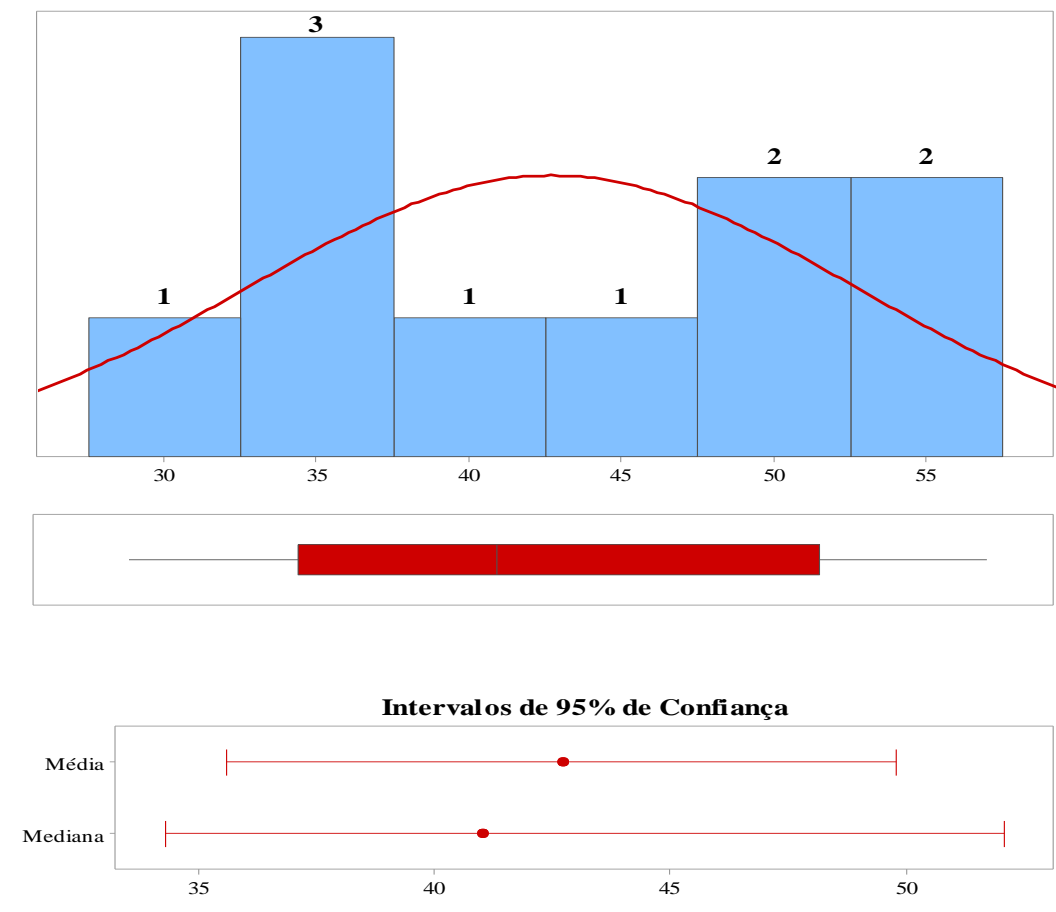

\begin{tabular}{|c|c|}
\hline \multicolumn{2}{|c|}{ Teste de normalidade } \\
\hline $\begin{array}{l}\text { A-Quadrado } \\
\text { Valor-p }\end{array}$ & $\begin{array}{r}0,31 \\
0,488\end{array}$ \\
\hline $\begin{array}{l}\text { Média } \\
\text { DesvPad } \\
\text { N }\end{array}$ & $\begin{array}{r}42,700 \\
9,911 \\
10\end{array}$ \\
\hline $\begin{array}{l}\text { Mínimo } \\
1^{\circ} \text {. Quartil } \\
\text { Mediana } \\
3^{\circ} \text { Quartil } \\
\text { Máximo }\end{array}$ & $\begin{array}{l}29,000 \\
34,500 \\
41,000 \\
51,500 \\
57,000\end{array}$ \\
\hline \multicolumn{2}{|c|}{ IC $95 \%$ - Média } \\
\hline \multicolumn{2}{|c|}{ IC $95 \%$ - Mediana } \\
\hline 34,315 & 52,054 \\
\hline \multicolumn{2}{|c|}{ IC $95 \%$ - DP } \\
\hline 6,817 & 18,094 \\
\hline
\end{tabular}

Fonte: SILVA FCC, et al., 2020. 
A Tabela 2 mostra os aspectos clínicos dos pacientes em tratamento em um Centro de Atenção Psicossocial (CAPS) do sudeste do Pará. Verifica-se que 4 (40,0\%) foram diagnosticados com depressão, 4 $(40,0 \%)$ fazem tratamento no CAPS pelo tempo de 6 a 10 anos, a maioria toma remédios controlados receitados pelo psiquiatra $(9 ; 90 \%)$, todos frequentam o CAPS 3 vezes por semana $(10 ; 100 \%)$, e a maioria não possui outro problema de saúde $(6 ; 60 \%)$ (Tabela 2).

Tabela 2 - Aspectos Clínicos dos pacientes em tratamento em um Centro de Atenção Psicossocial (CAPS) do sudeste do Pará.

\begin{tabular}{|c|c|c|c|}
\hline Aspectos Clínicos & Quantidade & Percentual (\%) & P-Valor(1) \\
\hline \multicolumn{4}{|l|}{ Qual o seu diagnóstico? } \\
\hline Depressão & 4 & $40,0 \%$ & \multirow{6}{*}{$0.0421^{*}$} \\
\hline Síndrome do Pânico & 1 & $10,0 \%$ & \\
\hline Transtorno de Ansiedade & 1 & $10,0 \%$ & \\
\hline Esquizofrenia & 1 & $10,0 \%$ & \\
\hline Não Sei & 4 & $40,0 \%$ & \\
\hline Transtorno Bipolar & 2 & $20,0 \%$ & \\
\hline \multicolumn{4}{|c|}{ Há quanto tempo você faz tratamento no CAPS? } \\
\hline Até 1 ano & 3 & $30,0 \%$ & \multirow{4}{*}{$0.0351^{*}$} \\
\hline 2 a 5 anos & 1 & $10,0 \%$ & \\
\hline 6 a 10 anos & 4 & $40,0 \%$ & \\
\hline 11 ou mais & 2 & $20,0 \%$ & \\
\hline \multicolumn{4}{|c|}{ Você toma remédios controlados receitados pelo psiquiatra? } \\
\hline Não & 1 & $10,0 \%$ & \multirow{2}{*}{$0.0001^{*}$} \\
\hline Sim & 9 & $90,0 \%$ & \\
\hline \multicolumn{4}{|c|}{ Com que frequência você vai ao CAPS? } \\
\hline 3 Vezes Por Semana & 10 & $100,0 \%$ & - \\
\hline \multicolumn{4}{|c|}{ Possui outro problema se saúde? } \\
\hline Diabetes & 2 & $20,0 \%$ & \multirow{3}{*}{$0.0241^{*}$} \\
\hline Hipertensão & 2 & $20,0 \%$ & \\
\hline Não & 6 & $60,0 \%$ & \\
\hline
\end{tabular}

Legenda: (1) Teste Qui-quadrado de Pearson (p-valor <0.05). ${ }^{*}$ Valores Significativos; NS Valores Não Significativos. $\mathbf{H}_{0}$ : Não há tendência significativa entre as frequências observadas $(p>0.05) . \mathbf{H}_{1}$ : Há tendência significativa entre as frequências observadas $(p<0.05)$.

Fonte: SILVA FCC. et al., 2020.

Quanto a verificação do nível de ansiedade dos participantes antes e depois da intervenção, observa-se na tabela 3 que houve diminuição significativa $(\mathrm{p}=0.0001)$ no escore médio dos pacientes submetidos a intervenção de musicoterapia, de forma que antes da intervenção a média obtida foi 48,60 , indicando elevado grau de ansiedade, enquanto que após 15 sessões a média obtida foi 39,30 indicando um nível baixo de ansiedade (Tabela 3).

Tabela 3 - Nível de ansiedade dos pacientes em tratamento em um Centro de Atenção Psicossocial (CAPS) do sudeste do Pará, antes e após a intervenção de musicoterapia.

\begin{tabular}{ccccccc}
\hline Momento da aplicação do IDATE & Média & DP & CV (\%) & Mín. & Med. & Máx. \\
\hline Antes & 48,60 & 17,93 & 36,89 & 23,00 & 50,00 & 70,00 \\
\hline Imediato (após uma sessão) & 40,50 & 14,22 & 35,12 & 22,00 & 44,00 & 60,00 \\
\hline Imediato (após 15 sessões) & 39,30 & 14,46 & 36,80 & 20,00 & 45,50 & 57,00 \\
\hline P-Valor & \multicolumn{7}{c}{$0.0001^{*}$}
\end{tabular}

Legenda: (1) Teste $t$ de Student para comparação de médias (p-valor $<0.05)$. *Valores Significativos; NS Valores Não Significativos. $\mathbf{H}_{0}$ : Não há diferença significativa entre as médias observadas $(p>0.05)$. $\mathbf{H}_{1}$ : Há diferença significativa entre as médias observadas $(p<0.05)$.

Fonte: Silva FCC, et al., 2020. 


\section{DISCUSSÃO}

A definição do repertório é considerada uma tarefa de grande responsabilidade, uma vez que a escolha inapropriada das músicas pode provocar efeitos negativos como o estresse e a ansiedade (GATTINO GS, 2016; BATISTA NS e RIBEIRO MC, 2016). Neste estudo, uma dificuldade encontrada foi a escassez de referencial teórico metodológico com critérios para a seleção das músicas. Desta forma, conforme critérios de Torchi TS e Barbosa MAM (2006), 6 (seis) músicas foram selecionadas e 4 foram compostas em parceria com a psicóloga e com a coordenadora do CAPS.

Durante a realização da intervenção, observou-se que os pacientes interagiam e cantavam mais quando as músicas apresentavam refrão curto e melodias de intensidade suave. De fato, de acordo com Nunes-Silva M, et al. (2016), músicas que apresentam ritmos lentos, melodias com intensidade suave e tons graves, promovem sensação de tranquilidade e conforto, e ainda possibilitam a redução das frequências respiratória e cardíaca.

Os resultados da caracterização da amostra expressam que a faixa etária dos participantes da pesquisa predominante é de $29-36$ anos (40\%). Em outros estudos realizados com pacientes em tratamento em Centros de Atenção Psicossociais, obteve-se resultados semelhantes, sendo evidenciada maior proporção de pessoas adultas jovens. A faixa etária predominante constatada por Cruz LS, et al. (2016) foi de 30 a 39 anos; a descrita por Peixoto FMS, et al. (2017) foi de 18 a 40 anos; e por Campos IO, et al. (2017) foi de 35 a 44 anos.

De acordo com Senicato C, et al. (2018), esses grupos etários encontrados nos estudos apresentam maior vulnerabilidade quanto a transtornos psiquiátricos, isolamento social, dificuldades econômicas, comorbidades e maior chance de estresse provocado pelo envolvimento em atividades de vida produtiva. Oliveira VC, et al. (2017) apontam para a vulnerabilidade dessas pessoas e ressaltam que os transtornos mentais atingem de forma predominante indivíduos que trabalham ou que estão em busca de um trabalho, incluídas no grupo etário denominado População Economicamente Ativa (PEA). Considerando que os transtornos mentais afetam a produtividade, é imprescindível que essas pessoas recebam suporte e tratamento (LEONARDO BC, et al., 2017).

Quanto ao gênero, nesse estudo foi evidenciada a prevalência do sexo feminino (70,0\%), bem como nos estudos realizados por Oliveira VC, et al. (2017), Cruz LS, et al. (2016), Campos IO, et al. (2017) e Leonardo BC, et al. (2017). De um modo geral, os homens procuram os serviços de saúde com menor frequência que as mulheres, e no que tange a procura por assistência psicológica, a menor demanda masculina pode estar ligada ao modelo de masculinidade e ao medo descobrir estar com a saúde mental afetada (CAMPOS IO, et al., 2017).

Em contrapartida, Senicato C, et al. (2018) ressaltam que a prevalência de mulheres com transtornos mentais pode estar relacionada a condições únicas da mulher como a gravidez e o puerpério, altos índices de violência física, sexual e psicológica existente no país, e ainda a opressão sofrida na dinâmica dos gêneros nas relações de poder. Para Leonardo BC, et al. (2017), fatores como a sobrecarga gerada pelo trabalho e pela responsabilidade familiar, além da renúncia do autocuidado em detrimento do cuidar do próximo, podem gerar frustações, angústia e ansiedade, contribuindo para o desenvolvimento de transtornos mentais.

No que se refere à escolaridade, constatou-se maior percentual de pessoas que possuem ensino fundamental incompleto (30\%) e ensino médio completo (30\%). Oliveira VC, et al. (2017) e Cruz LS, et al. (2016) também obtiveram resultados similares, com a maior proporção de pessoas com ensino fundamental incompleto e ensino médio concluído.

Leonardo BC, et al. (2017) destacam que quanto menor a escolaridade maior a chance do desenvolvimento de transtornos mentais, uma vez que essas pessoas podem apresentar dificuldade de conseguir um emprego, baixa remuneração e sentindo-se, por consequência, pouco valorizadas.

No que concerne ao estado civil, $60 \%$ dos participantes desse estudo declararam-se solteiros. Peixoto FMS, et al. (2017) também constataram proporção elevada de pessoas solteiras em suas pesquisas, com os percentuais próximos de $50 \%$, e acreditam que esses resultados estão relacionados ao estado de 
adoecimento mental pela dificuldade de iniciar um novo relacionamento. Senicato C, et al. (2018) ressaltam como fator contributivo para a elevada porcentagem de solteiros, a possibilidade dessas pessoas apresentarem dificuldade em estabelecer um relacionamento estável e possuírem menor chance de se casar.

Referente aos aspectos clínicos, o diagnóstico com maior percentual verificado foi a depressão (40\%). 0 resultado da prevalência de mulheres com depressão é ratificado por Gonçalves AMC, et al. (2018) que ressaltam o acometimento aproximadamente duas vezes maior em mulheres que em homens devido a fatores como diferenças fisiológicas e hormonais, baixa renda, baixo nível de escolaridade, questões socioculturais e diferentes formas de lidar com situações que causam estresse.

O tempo de tratamento predominante entre os participantes foi de 6 a 10 anos (40\%). Leonardo BC, et al. (2017) ressaltou que os transtornos mentais tendem a ser crônicos e incapacitantes, fazendo o indivíduo perder anos potenciais de vida.

A maioria dos participantes desse estudo toma remédios controlados receitados pelo psiquiatra (90\%). De acordo com Alcântara CB, et al. (2018) a terapia medicamentosa visa a redução dos sintomas provocados pelo transtorno mental a fim de favorecer ao indivíduo a melhor adaptação à realidade possível, sendo assim, é considerada um importante recurso no tratamento em saúde mental.

Todos os participantes frequentam o CAPS 3 vezes por semana, demonstrando que os participantes desse estudo estão em tratamento semi-intensivo (BRASIL, 2004).

As comorbidades mais frequentes foram diabetes (20\%) e hipertensão (20\%). Braga DC, et al. (2017) demonstraram que pessoas com diabetes podem ter os estados emocionais negativos induzidos pela hipoglicemia e hiperglicemia graves. Em sua pesquisa, 465 pessoas autorreferidas diabéticas apresentavam depressão, sendo 79,52\% do sexo feminino. Quanto à hipertensão, Li Z, et al. (2015) ressaltam que é comum pacientes hipertensos apresentarem depressão, encontrando $21,3 \%$ de hipertensos com sintomas depressivos em sua pesquisa.

Quanto ao nível de ansiedade, obteve-se uma redução significativa no escore médio dos pacientes submetidos à intervenção musical, que passou de 48,60 para $39,30(p=0.0001)$. Firmeza MA, et al. (2017) revelaram que $85 \%$ dos pacientes apresentaram ansiedade moderada antes da intervenção, e $55 \%$ apresentaram nível de ansiedade baixa após a intervenção. Houve ainda diminuição média de 10,95 mmHg na pressão arterial sistólica, 3,95 $\mathrm{mmHg}$ na pressão arterial diastólica, 7,50 batimentos por minuto na pulsação e 2,25 inspirações por minuto na frequência respiratória. Resultados similares foram encontrados nas análises qualitativas de músicas compostas para indução de relaxamento, com diminuição de valências negativas, como "raiva/medo" e excitabilidade alta, favorecendo à promoção de relaxamento, com consequente redução da ansiedade, por meio de intervenção musical (NUNES-SILVA M, et al., 2016).

O potencial ansiolítico da música está relacionado ao prazer e pode ser justificado pela ativação de áreas do cérebro como as vias dopaminérgicas, que gera respostas positivas de esforço e recompensa, reduzindo a ansiedade, promovendo o relaxamento muscular e por vezes possui melhor eficácia que fármacos como midazolam e benzodiazepina (ARAÚJO TC e SILVA LWS, 2013; AREIAS JC, 2016; FIRMEZA MA, et al., 2017).

Souza J, et al. (2019) constatou a redução da ansiedade por meio do canto coral, que possibilitou ainda a integração entre os participantes. Nascimento E, et al. (2018) ratificou a capacidade que a música apresenta de integrar as pessoas e ainda destacou a melhora da autoestima e seu funcionamento como um meio importante de comunicação. Já Batista EC, et al. (2018) evidenciaram a música como fator contributivo para um atendimento humanizado e estabelecimento de vínculos entre usuários e profissionais.

Nessa pesquisa foi incentivado que os participantes cantassem durante a sessões com música, o que possibilitou maior integração tanto entre ele quanto com os profissionais de saúde que estavam presentes, que também cantaram e estavam sempre dispostos a ajudar. De acordo com Souza J, et al. (2019), a música promove reflexões acerca das letras entoadas, o que foi corroborado nessa pesquisa, por meio da explicação dos significados das músicas aos participantes e abertura de espaço para que os mesmos expressassem suas opiniões e experiências de vida. 


\section{CONCLUSÃO}

Apesar do número amostral reduzido, a maior proporção dos participantes está na faixa etária de 29 a 36 anos, são do sexo feminino, solteiros(as), tem depressão, tomam medicamento controlado e estão em tratamento no CAPS há mais de 6 anos. O repertório de músicas com mensagens positivas, melodias de intensidade suave e com refrão curto, permite maior interação do público alvo. Quanto ao nível de ansiedade, a intervenção musical pode auxiliar na diminuição da ansiedade de modo significativo. Este estudo é pioneiro no CAPS em questão e a música deve ser estimulada em outros centros de atenção psicossocial.

\section{REFERÊNCIAS}

1. ALCÂNTARA CB et al. A terapêutica medicamentosa às pessoas com transtorno mental na visão de profissionais da enfermagem. Esc. Anna Nery, 2018; 22(2): e20170294.

2. ALMEIDA AS, SILVA MR. Os efeitos das atividades musicais como modalidade alternativa de cuidado em saúde mental. Rev. enferm. atenção saúde, 2013; 2(1): 13-20, 2013.

3. ARAÚJO TC, SILVA LWS. Música: estratégia cuidativa para pacientes internados em unidade de terapia intensiva. Rev enferm UFPE online, 2013; 7(5): 1319-1325.

4. AREIAS JC. A música, a saúde e o bem estar. Nascer e Crescer, 2016; 25(1): 7-10

5. BATISTA NS, RIBEIRO MC. O uso da música como recurso terapêutico em saúde mental. Rev. Ter. Ocup. Univ. São Paulo (Online), $2016 ; 27(3): 336-41$.

6. BATISTA EC et al. Viver e não ter a vergonha de ser feliz: Música e produção de sentidos num CAPS da Amazônia Ocidental. PSI UNISC, 2018; 2(2):162-176.

7. BOTELHO JV, LIMA MV. Percepção das emoções dos usuários do CAPS II: um relato de experiência. Fractal, Rev. Psicol., 2015; 27(2): 160-164.

8. BRASIL. Ministério da Saúde. Secretaria de Atenção à Saúde. Departamento de Ações Programáticas Estratégicas. Saúde mental no SUS: os centros de atenção psicossocial. Brasília: Ministério da Saúde, 2004; 86 p.

9. BRAGA DC et al. Fatores associados à depressão em indivíduos com diabetes mellitus. Arq. Catarin Med., 2017; 46(3): 118128.

10. BORINE MS. Ansiedade, neuroticismo e suporte familiar: evidências de validade do Inventário de Ansiedade Traço-Estado (IDATE). Tese (Doutorado) - Programa de Pós graduação Stricto sensu em Psicologia. Universidade São Francisco, Itatiba, $2011 ; 122 p$.

11. CAMPOS IO, et al. Saúde mental e gênero: O perfil sociodemográfico de pacientes em um centro de atenção psicossocial. Estudos de Psicologia, 2017; 22(1): 68-77.

12. CASTILLO ARG, et al. Transtornos de ansiedade. Rev. Bras. Psiquiatr., 2000; 22( Suppl 2 ): 20-23.

13. CHAN MF, et al. Effects of music on depression in older people: a randomised controlled trial. J Clin Nurs, 2012; 21(5-6): 776-783.

14. CRUZ LS, et al. Perfil de pacientes com transtornos mentais atendidos no centro de atenção psicossocial do Município de Candeias: Bahia. Rev. bras. ciênc. saúde, 2016; 20(2):93-98.

15. ESPERIDIÃO E, et al. A Enfermagem Psiquiátrica , a ABEn e o Departamento Científico de Enfermagem Psiquiátrica e Saúde Mental : avanços e desafios. Rev. bras. enferm., 2013; 66(spe): 171-176.

16. FIRMEZA MÁ, et al. Uso da música no controle da ansiedade em ambulatório de cabeça e pescoço: ensaio clínico randomizado. Rev. esc. enferm. USP, 2017; 51: e03201.

17. FRANZOI MAH, et al. Intervenção musical como estratégia de cuidado de enfermagem a crianças com transtorno do espectro do autismo em um centro de atenção psicossocial. Texto Contexto Enferm., 2016; 25(1): e1020015.

18. GATTINO GS. Algumas considerações sobre os efeitos negativos da música. Hodie, 2016; 15(2).

19. GONÇALVES AMC, et al. Prevalência de depressão e fatores associados em mulheres atendidas pela Estratégia de Saúde da Família. J. Bras. Psiquiatr., 2018; 67(2): 101-109.

20. LEONARDO BC, et al. Prevalência de transtornos mentais e utilização de psicofármacos em pacientes atendidos em um ambulatório médico de especialidades. Arq. Catarin Med., 2017; 46(2): 39-52.

21. LI Z, et al. Prevalence of depression in patients with hypertension: A systematic review and meta-analysis. Medicine (Baltimore), 2015; 94(31):e1317.

22. MENDES MF, et al. Saúde mental e arte: relato de uma oficina de experiências estéticas em um centro de atenção psicossocial. Cadernos Brasileiros de Saúde Mental, 2016; 8(20): 69-79.

23. MIRANDA MC, et al. La música como una herramienta terapéutica en medicina. Rev Chil Neuro-Psiquiatr, 2017; 55(4): 266277.

24. NASCIMENTO E., et al. Oficinas terapêuticas com música, em saúde mental. Revista Contexto \& Saúde, 2018;18(34): 1519.

25. NUNES-SILVA M, et al. Avaliação de Músicas Compostas para Indução de Relaxamento e de seus Efeitos Psicológicos. Psicologia: Ciência e Profissão, 2016; 36(3): 709-725.

26. OLIVEIRA VC, et al. Perfil sociodemográfico e clínico de pessoas atendidas em um CAPS AD do Sul do país. Rev baiana enferm., 2017; 31(1):e16350.

27. PEIXOTO FMS, et al. Perfil epidemiológico de usuários de um centro de atenção psicossocial em Pernambuco, Brasil. J Health Sci, 2017;19(2):114-9.

28. ROHR RV, ALVIM NAT. Intervenções de enfermagem com música: revisão integrativa da literatura. R de Pesq: cuidado é fundamental Online, 2016; 8(1):3832-3844.

29. SENICATO C, et al. Transtorno mental comum em mulheres adultas: identificando os segmentos mais vulneráveis. Ciênc. saúde coletiva, 2018; 23(8): 2543-2554.

30. SOUZA J, et al. A música como prática de promoção da saúde na adolescência. Rev. Enferm. UFSM., 2019; 9(11); 1-14.

31. TORCHI TS, BARBOSA MAM. A música como recurso no cuidar em enfermagem. Ensaios e Ciência, 2006; 3(10): $125-38$. 